\title{
Connectivity of snow particles
}

\author{
K. ITAGAKi AND G. E. LemieuX \\ U.S. Army Cold Regions Research and Engineering Laboratory, Hanover, NH 03755, U.S.A.
}

\begin{abstract}
An optical system, potentially suitable for quantitative characterization of snow, was conceived and tested. The system uses a laser beam to scan a thick section of snow impregnated with an opaque medium and observes light transmission through the slab.
\end{abstract}

\section{INTRODUCTION}

The physical properties of snow are a complex function of many parameters such as density, particle size, age and moisture content. Global structure and local texture are other important factors affecting mechanical, electrical, thermal, optical and other properties. Quantitative characterization of the structure and texture of snow is difficult.

The structure and the texture are the three-dimensional arrangement of the constitutent particles. An aggregate of crushed ice may have a different structure and texture from a naturally occurring snow pack, even though the density, particle size and some other parameters are the same.

Several efforts have been made to collect numerical information about the snow pack, such as the form factor (Yosida and Kuroiwa, 1951), the coordination number (Sato and Tusima, 1989), structural factors (Kuroiwa, 1975) and a set of 21 parameters (point, stereological, geometrical, crystallographical and topological) (Good, 1975). However, those efforts tended to concentrate on local, individual snow grains, and did not consider connection in the global context. The question here is: how can we describe snow in a quantitative way?

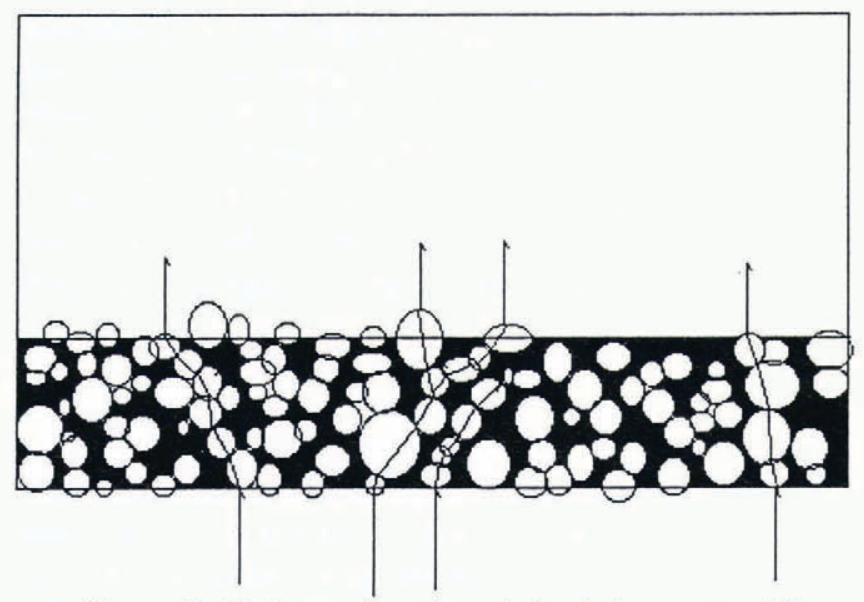

Figure 1. Light passing through bonded snow particles surrounded by an opaque medium.
The way that snow particles in the snow pack are connected to each other is essential information. Several approaches can be made to study this connectivity. The straightforward approach is by making consecutive thin sections and then reconstructing them. This is a very time-consuming and tedious process. Some efforts have been made to reconstruct thin sections (Huse and others, 1985), but we still do not have a good way to present the results.

The theory of fractals has been successfully used to analyze colloidal aggregates (e.g. Schaefer and Keefer, 1986), atmospheric turbulence and rainfall distribution (Schertzer and Lovejoy, 1987). It seems a good starting point, although application to snow may need some more development. However, the quantitative characterization of snow may have to take such a route.

\section{EXPERIMENTAL METHOD}

One way to gain information on snow connectivity is to immerse a snow sample in an opaque medium and scan it, as shown in Figure 1. Since snow particles are transparent, light can be transmitted through the bonds of the connected grains, giving us information about connectivity. The thicker the sample, the less light emerges, and the deflection of light from the entry point gives us further information (Fig. 1).

Two types of snow and a model snow made of glass beads were tested. Samples made from fresh, natural snow and from sieved snow samples were impregnated by a dimethyl phthalate solution of methylene blue. The melting point of dimethyl phthalate is $2^{\circ} \mathrm{C}$, but it stays super-cooled long enough for us to inject it into the snow structure before it solidified. The methylene blue effectively absorbs red $\mathrm{He}-\mathrm{Ne}$ laser light. After the solution solidified, the snow sample was mounted on a glass plate and microtomed to the desired thickness.

Glass beads were used to make a model of uniform, transparent spheres. The beads were stacked, layer by layer, using clear spray paint to stick the layers together. First, clear paint was sprayed on a slide glass $(50 \times$ $75 \mathrm{~mm}$ ) and the glass beads were sprinkled uniformly to 


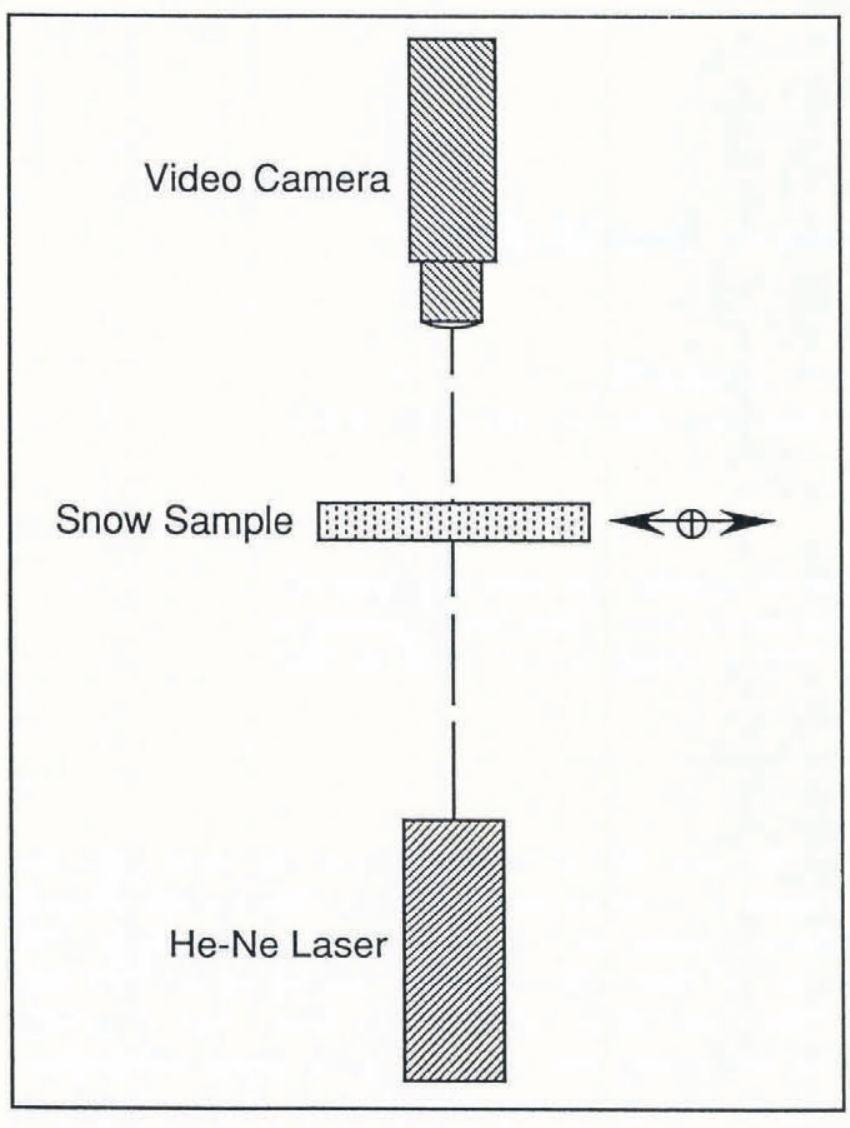

Figure 2. Schematic of apparatus.

form a single layer. After the paint dried, the second layer was formed over the previous layer in the same way. A $10 \mathrm{~mm}$-wide strip was covered by a shutter so that no additional layers could be built over that part of the sample. The coverage was increased by $10 \mathrm{~mm}$ at each step, so that five layers of $10 \mathrm{~mm}$-wide strips were built. The model snow pack was immersed in an opaque fluid (Japanese Sumi ink).

Both the real and model snow pack were scanned by a narrow $(0.5 \mathrm{~mm})$ laser beam. The positions of the laser and the video camera were fixed, anchoring the point of beam entry relative to the camera. Deviation of the beam can be readily measured on the video screen. Scanning was done by an $x-y$ positioner moving in the $x$ and $y$ directions perpendicular to the He-Ne laser beam (Fig. 2). Transmitted light was observed by the video camera and recorded on videotape.

The transmitted light emerged as bright spots and diffused specks, usually slightly off the beam (1-2 mm), indicating that the light traversed tortuous paths. The bright spots indicate that light traversed with little absorption, probably taking a straight course or being totally reflected at interfaces between a snow grain and the immersing medium. Those bright spots are usually recorded in $5-7$ video frames, over $\sim 0.2 \mathrm{~s}$. Since the scan rate was $1 \mathrm{~mm} \mathrm{~s}^{-1}$, such bright spots were $\sim 0.2 \mathrm{~mm}$, which is smaller than either the beam size of $\sim 0.5 \mathrm{~mm}$ or the grain size. Some preferential relationship between the laser beam and connected snow grains seems likely to have caused the bright spots.

When the light scattered inside the snow grains, the whole grain was illuminated with reduced intensity, causing the diffuse specks. The light emerging after a long, tortuous pass would have endured heavy absorption. The reduction of light is considerable, so that detection of diffuse spots on a thick sample depends on the sensitivity of the camera and background noise.

In addition to laser scanning, in some cases the top side of the samples was illuminated by green filtered light. The top illumination helps to identify the grain where light emerged, as well as snow grain size. Such information is useful to our study of the structure of snow samples.

A total of five sets of three forward and reverse scans covered a $75 \times 50 \mathrm{~mm}$ area and constituted one set of scans. This scan pattern was designed to scan the five different layers of glass beads in the model snow and was also found to fit well to the real snow. A slow scanning rate of $1 \mathrm{~mm} \mathrm{~s}^{-1}$ was chosen to catch any emergence of light between video frames. Since the light beam tended to be deflected within the connected grains, and the deflection provided us with more information on connectivity of grains, video recording is well suited for these studies. However, automation of data analysis may be more difficult than simple light intensity measurements.

The emergence of light was observed on a TV monitor. Time marks of each emergence of light were manually recorded, with the elapsed time from the beginning of the scan calculated. The number of intervals in which emergence was recorded was counted.

\section{DATA ANALYSIS AND RESULTS}

The separation of each scan by $1 \mathrm{~mm}$ provided little overlap, so that scans of snow by the present setup can be considered a one-dimensional cross-section of the light transmission properties of snow. Scans were made at a constant speed and the time marks recorded on the videotapes provided us with an excellent measure of distances scanned. The recorded time mark can show the location of light emergence.

There are many possible ways to analyze the video images of scans on the tapes. Because snow could be viewed as a bonded percolation aggregate above criticality, functional box counting was tried: it is simple and does not require elaborate equipment or software. The functional box counting method can also provide insight into the multi-fractal nature of snow. The percolation aggregate would show fractal properties within a certain scale range. All snow particles on the surface have to be supported by bottom particles, unlike the situation with a colloidal suspension, and the percolation aggregate has to be above criticality. However, many grains on the surface showed no light emergence. No observable light emergence indicates heavy light absorption; probably, those grains are connected to the bottom grains through tortuous paths.

The box counting was made by successively doubling the interval (box) from 2 to $512 \mathrm{~s}$ (corresponding to 2 to $512 \mathrm{~mm}$ ) and counting the number of boxes in which light emergence was observed. Log of interval vs log of counts would give us a linear relationship if scaling is invariant, and its slope indicates the fractal dimension at the thickness. As the thickness is reduced, the probability of 


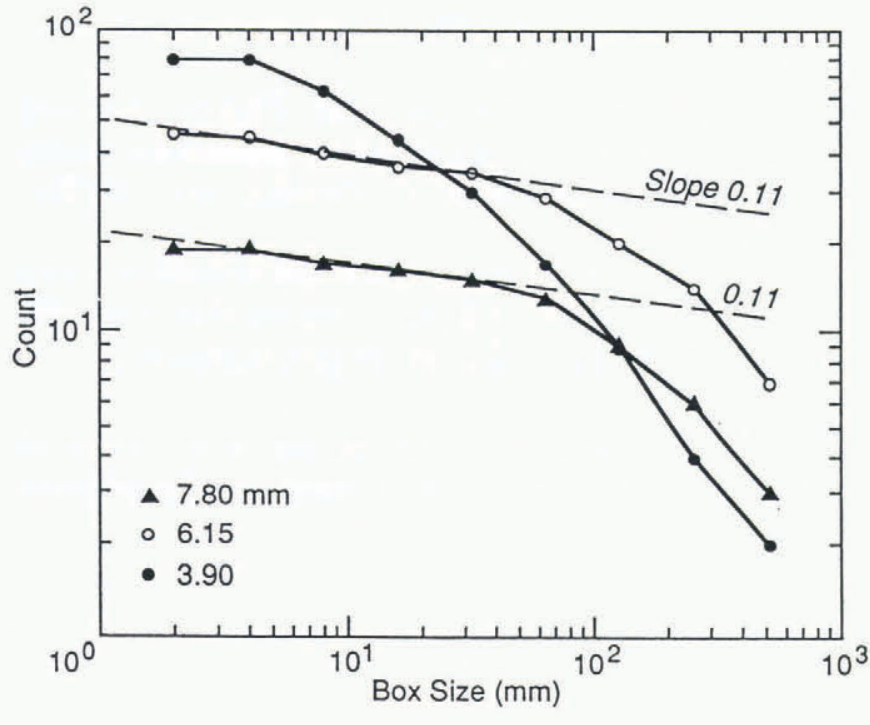

Figure 3. Log of box size vs log of number of boxes having light emergence. Fractal structure is indicated by the linear portion of the curves. The thin $(3.9 \mathrm{~mm})$ sample had no linear part because of resolution limits. The end parts of the larger box count curves tended to have a slope of 1 owing to homogeneity in the box size range.

light passing through the snow particles and being detected increases. Figure 3 shows an example of plots using an aggregate of sieved snow particles having sizes less than $1 \mathrm{~mm}$.

Curves of $\log$ of box size vs log of the number of boxes having light emergence for 6.15 and $7.8 \mathrm{~mm}$-thick samples show a linear part, both having the same slope of 0.11 for box sizes below $64 \mathrm{~mm}$. Those slopes correspond to the fractal dimensions of 0.11 . The curve for the $3.9 \mathrm{~mm}$-thick sample had no linear portion. Apparently, interaction between the smaller box size and the larger box size overlapped. The fractal dimension of snow samples obtained by box counting as a function of thickness within the thickness range changed little.

As mentioned before, box sizes larger than $64 \mathrm{~mm}$ tended to have a slope of 1 . This trend can be expected, since larger boxes tended to contain at least one light emergence. In other words, the snow sample that is larger than $64 \mathrm{~mm}$ can be treated as a uniform mass. Box sizes smaller than $4 \mathrm{~mm}$ tended to saturate owing to the limited number of data and the resolution of the measurement.

The probability of observing light emergence is the product of the probability of light illuminating the entrance of the conduit channel and the probability that the level of light absorption would be low enough so that observable light emerges. Since the bottom surface is undisturbed and scans were made on identical tracks, any change caused by progressively thinner samples is caused by the bulk of the snow. The count of light emergences doubled as the samples became $1.6 \mathrm{~mm}$ thinner, but there was no change in the slope.

The sensitivity of the video camera system used in this study limited detection of low-level light emergence from thick samples. Interestingly, detection by change in color with green illumination seems more sensitive than detection with only the laser. Apparently, some low- level cutoff in the video recording system reduced sensitivity in the monochromatic $\mathrm{He}-\mathrm{Ne}$ only. With green illumination, the higher base line enabled us to detect low-level red laser light.

\section{DISGUSSION}

A combination of off-the-shelf items-laser, video camera and recorder, and $x-y$ positioner-seems to show great potential for examining snow structure and texture. Several areas, however, still need improvement and development.

Monitoring the video display and manually recording light emergence is time consuming, and still misses some data. It is difficult to record manually many light emergences in short succession in thinner samples. Light intensity could not be accurately measured visually. The relative location of light emergence has not yet been attempted. Image analysis via computer can solve these problems but will require a computer with a large memory and sophisticated software. Other approaches are being tested.

The optical scanning method described above has some drawbacks. The intensity of light emerging from the tortuous paths may be too low to be detected. Also, light can be transmitted through a thin layer of the mixture (dimethyl phthalate solution of methylene blue), giving false data that are indistinguishable from light emerging from ice grains.

Other radiation could be adapted to scanning methods. For ultrasonic methods, either longitudinal or transversal waves are possible. Since transverse waves cannot be transmitted in a fluid, the connectivity of solid particles such as snow could be better studied. However, ultrasonic scanning needs a coupling fluid. Scanning with a thin layer of fluid between the ultrasonic transducer and the delicate structure of snow may prove a difficult problem.

Ultrasonic and X-ray scattering and absorption methods have been used in the characterization of colloids. The same technique could be applicable to snow characterization. This approach would not need much development, but merely adjustment of the frequency and selection of the proper medium.

To be able to use these methods fully in the study of snow strength and other properties, we need a fundamental scheme for quantitative snow characterization. Connectivity and coordination number are closely related; they are also related to density and strength of snow. The techniques described above, together with our laser scan method, could yield numerical information useful for characterizing snow.

\section{REFERENCES}

Good, W. 1975. Numerical parameters to identify snow structure. International Association of Hydrological Sciences Publication 114 (Symposium at Grindelwald 1974 Snow Mechanics), 91-102.

Huse, J., K. Tusima and K. Tusima. 1985. Three dimensional structure of snow by consecutive micro- 
toming and photographic re-construction technique. Preprint for Japanese Society of Snow and Ice 1985 meetings, 185. [In Japanese.]

Kuroiwa, D. 1975. Mechanics and structure of snow as a dispersed system. International Association of Hydrological Sciences Publication 114 (Symposium at Grindelwald 1974 - Snow Mechanics), 3-15.

Sato, H. and K. Tusima. 1989. Stereographic observation of thick snow section samples. Preprint for Japanese Society of Snow and Ice 1989 meetings, 100. [In Japanese.]

Schaefer, D. W. and K.D. Keefer. 1986. Structure of random silicates: polymers, colloids, and porous solids.
In Pietronero, L., ed. Fractals in physics. Amsterdam, North-Holland, 39-45.

Schertzer, D. and S. Lovejoy. 1987. Physical modeling and analysis of rain and clouds by anisotropic scaling multiplicative processes. J. Geophys. Res., 92(D8), 9693-9714.

Yosida, Z. and D. Kuroiwa. 1951. On a number which determines the shape of snow particles. Low Temp. Sci. 7, 43-50. [In Japanese with English summary.]

The accuracy of references in the text and in this list is the responsibility of the authors, to whom queries should be addressed. 